\title{
Addition Coupled Electron Transfer (ACET) and Addition Coupled Proton Coupled Electron Transfer (ACPCET)
}

Yumiao $\mathrm{Ma}^{* a, \mathrm{~b}}$

a. BSJ Institute, Haidian, Beijing, 100084, People's Republic of China. ymma@bsj-institute.top

b. Hangzhou Yanqu Information Technology Co., Ltd. Xihu District, Hangzhou City, Zhejiang Province, 310003, People's Republic of China

\begin{abstract}
The new types of elementary reaction in which a nucleophilic addition (A) to quinones is coupled with electron transfer (ET) and even further proton transfer (PT) are suggested herein by density functional theory calculation, which are called Addition Coupled Electron Transfer (ACET) and Addition Coupled Proton Coupled Electron Transfer (ACPCET). With a [2.2]paracyclophanederived biquinone as the substrate, the nature of nucleophilic addition onto its $\mathrm{sp}^{2}$ carbons exhibits a change from stepwise A-ET-PT to ACET-PT and further to ACPCET, in parallel with the decreased nucleophilicity of the attacking reagent. In addition, we further proposed six possible potential energy surfaces and the coupling modes among A, ET and PT, among which three have been found in this work. Quasi-classical trajectory shows that the ACET and PT event can also be dynamically concerted even for an ACET-PT mechanism.
\end{abstract}

\section{Introduction}

Since its first being proposed in $1981^{1}$, the proton coupled electron transfer (PCET) has become a well-known concept, and both its theory ${ }^{2-9}$ and practical use ${ }^{10-16}$ have been continuously explored. Under this scheme, besides the Hydrogen Atom Transfer (HAT) reaction, in which both the proton and electron being transferred involves the same orbital, during the reaction of a hydrogen donor $(\mathrm{AH})$ and an acceptor $(\mathrm{B})$ to give $\mathrm{A}$ and $\mathrm{BH}$, there are three possible modes: stepwise electron transfer (ET) to form $\mathrm{AH}^{+}$and $\mathrm{B}^{-}$followed by a proton transfer (PT); stepwise $\mathrm{PT}$ affording $\mathrm{A}^{-}$and $\mathrm{BH}^{+}$followed by an ET; and, as called PCET, a concerted proton-electron transfer in one elementary step, especially when single PT or ET is thermodynamically unfavorable. The key point of PCET is that PT and ET can interplay and promote each other: ET leads to increased acidity of the A-H bond, promoting the PT process; at the meantime the negative charge resulted by PT on A further promotes ET. The inter-promoting nature of PT and ET enables their coupling, causing the elementary step of PCET as a result.

The reaction mode possible to couple with ET is not limited to PT. In this work, we focus on another important elementary reaction, namely the nucleophilic addition to electron-deficient olefins (noted as A). The nucleophilic addition reaction towards an olefin substrate is, to some extent, similar to PT from an acidic substrate: they both create a partial negative charge which is prone to be oxidized, and both are activated by a more oxidized (electrophilic) substrate. With a [2.2]paracyclophane-derived biquinone compound $\mathbf{1}$ (Figure 1) as the model substrate, we studied the impact of nucleophile on the reaction mechanism, and found that the addition reaction can couple with ET and PT, leading to the new elementary reaction of Addition Coupled Electron Transfer (ACET) and Addition Coupled Proton Coupled Electron Transfer (ACPCET) ${ }^{17}$. 

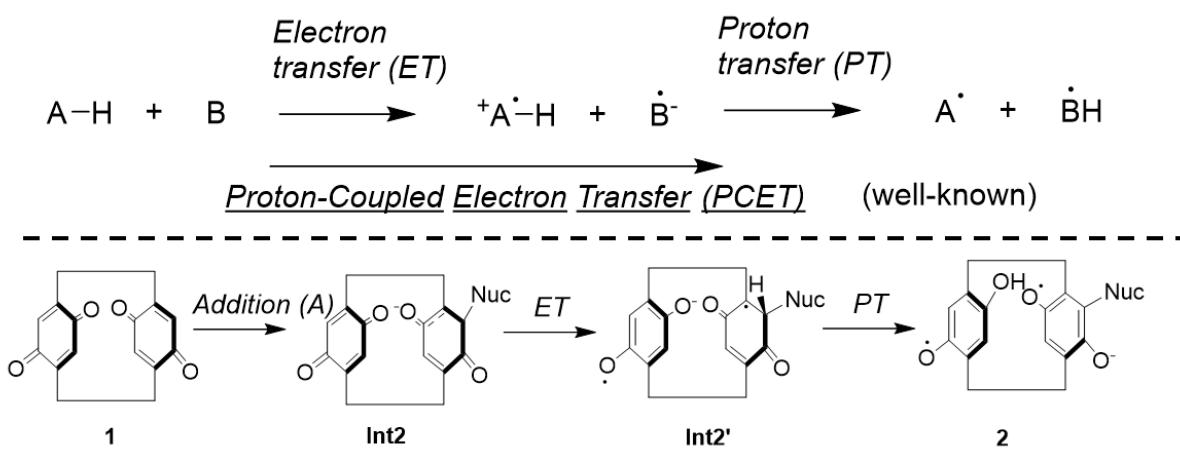

Do open-shelled intermediates exist in the addition pathway?

Do A, PT and ET happen in a stepwise or concerted manner?

Is a stepwise mechanism dynamically concerted?

Addition-Coupled ET (ACET) I Addition-Coupled PCET

$\underline{(A C P C E T)}:$ Do they exist?

Figure 1. A summary of the concept of PCET, ACET and ACPCET.

\section{Results and Discussions}

\section{Initial Insight}

Compound 1 has been synthesized and characterized by Staab by oxidizing its phenol precursor in $1973^{18}$. It was chosen as the model substrate in this work, because it bears two quinone rings connected by two short and rigid $\left(\mathrm{CH}_{2}\right)_{2}$ linkers, enabling short contact of the two reactive rings. As a result, it is of concern that once a nucleophile attacks one quinone ring, leading to an enolate (Int2 in Figure 1) whether the other quinone ring could act as an intramolecular oxidant to achieve an open-shelled compound Int2'. Furthermore, the delicate structure of 1 enables an intramolecular PT from Int2' across the two quinone rings, affording the final biradical product 2. Of interests in this paper is that whether these A, PT, ET processes can efficiently couple, both in terms of minimum energy reaction path (MEP) and reaction dynamics.

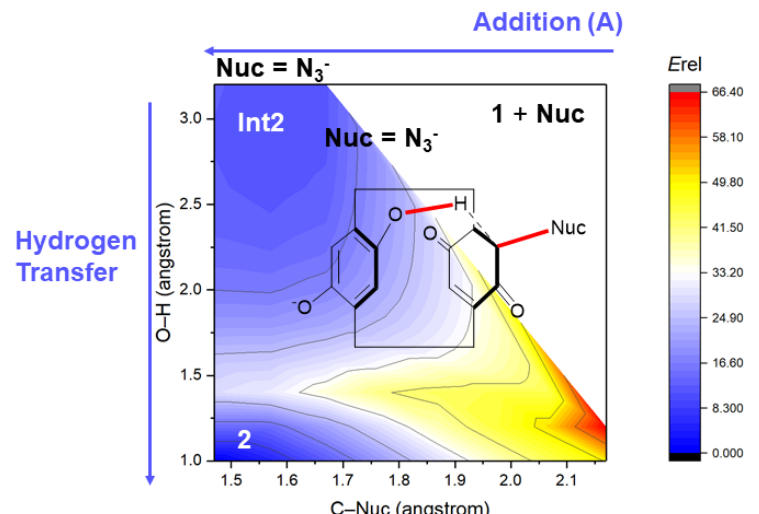

Addition product is close-shelled: A-ET-PT or A-PCET

Addition product is open-shelled: ACET-PT (stepwise or dynamically concerted)

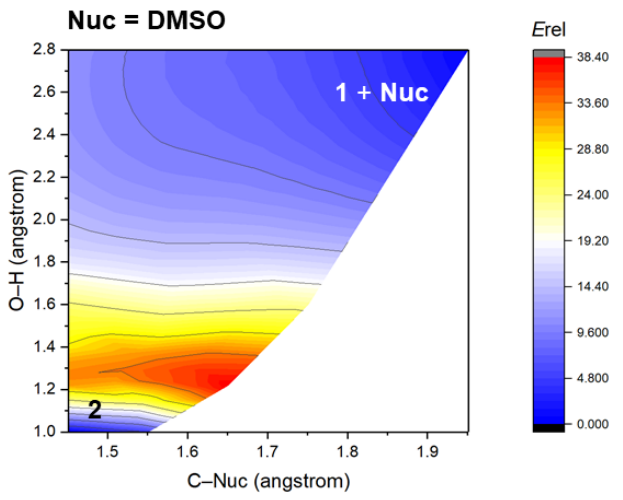

Int2 does not exist: ACPCET

Figure 2. The two-dimensional potential energy contours for the overall A-ET-PT reaction, with $\mathrm{N}_{3}{ }^{-}$ and DMSO as the nucleophile, respectively. Energies are shown in $\mathrm{kcal} / \mathrm{mol}$, and the interested bonds are labelled as red. 
The first problem of concern is whether the intermediates mentioned in Figure 1 can exist. The answer to this question depends on the nucleophilicity of the attacking reagent. Two typical twodimensional potential energy surfaces along the reaction coordinate of both addition and hydrogen transfer are shown in Figure 2. On the one hand, for a common nucleophile (Nuc) with enough nucleophilicity, the approaching to 1 leads to a minimum, corresponding to either close-shelled Int2 or open-shelled Int2'. In this case, we expect that a stepwise A-ET-PT or A-PCET or ACET-PT should happen. On the other hand, when the nucleophilicity of Nuc is extremely low, such as Nuc = DMSO, Int2 and Int2' are unable to be a minimum on the potential energy surface, and since $\mathbf{1}$ is thermodynamically unable to oxidize Nuc to initialize an ET-A-PT process (see Supporting Information for the redox thermodynamics), A, ET, PT have to be coupled in one single elementary step, which corresponds to the ACPCET mechanism. In the following part, we separately discuss the three cases: stepwise A-ET-PT; ACET-PT; ACPCET.

Stepwise A-ET-PT when $\mathrm{Nuc}=\mathrm{Na}(\mathrm{THF})_{2}(\mathrm{OMe})($ as noted by $\mathrm{NaOMe})$

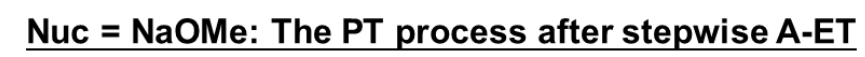

(a)

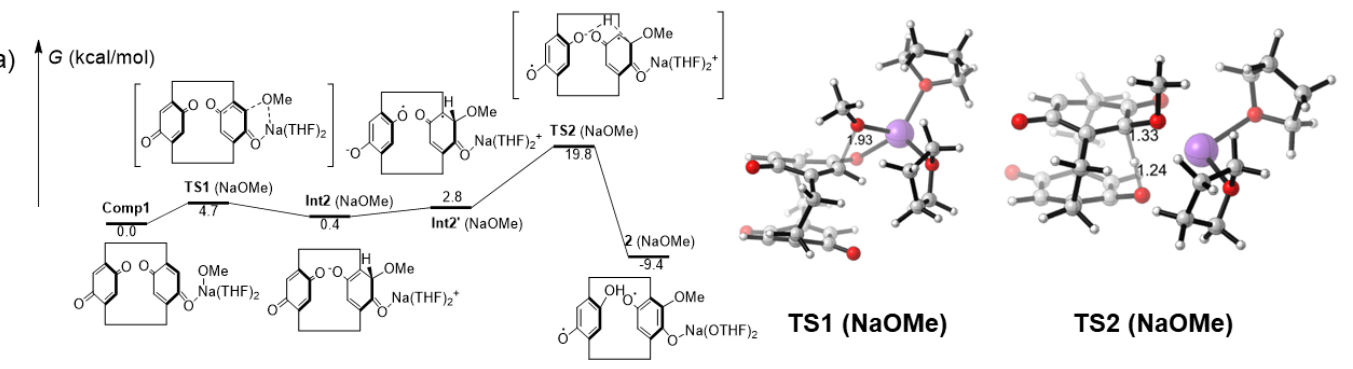

(b)
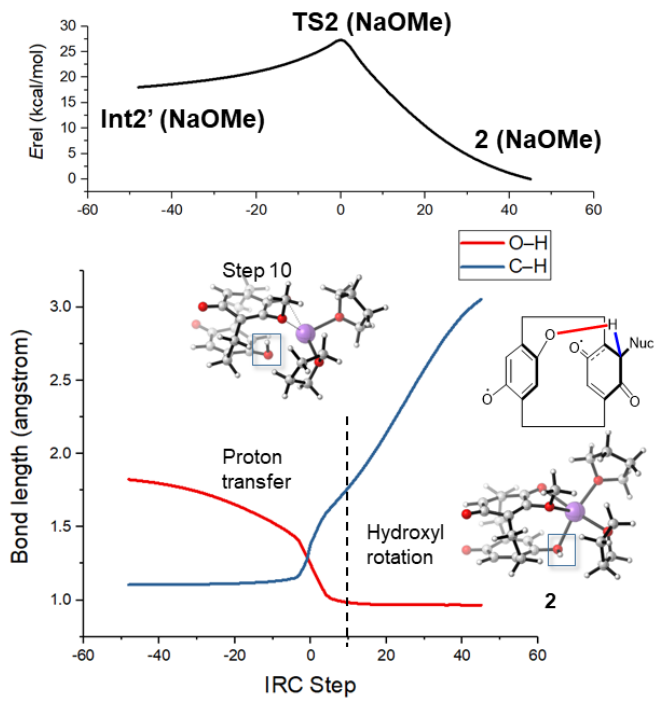

(c) Spin Density of Key Species Int2' (NaOMe)

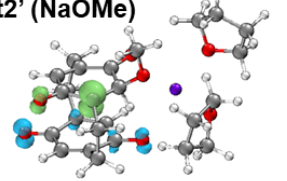
TS2 (NaOMe)
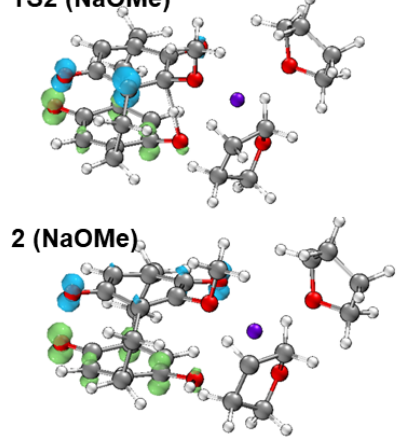

Figure 3. (a) The Gibbs free energy profile for the A-ET-PT reaction with $\mathrm{Na}(\mathrm{OMe})(\mathrm{THF})_{2}$ (noted as $\mathrm{NaOMe}$ ) as the nucleophile. Distances are in angstrom. (b) the evolution of energy and key bond lengths along the IRC of TS2 $(\mathrm{NaOMe})$. (c) The spin density isosurface of the key species.

With $\mathrm{NaOMe}$ as Nuc, all the intermediates and transition states in Figure 1 can be located, as shown in Figure 3a. The complex of $\mathbf{1}$ with $\mathrm{NaOMe}$ undergoes a rapid nucleophilic addition with a 
barrier of $4.7 \mathrm{kcal} / \mathrm{mol}$, affording a close-shelled enolate Int2 $(\mathrm{NaOMe})$. Then some geometry adjustment occurs, resulting in another minimum on the open-shelled potential energy surface, namely Int2'. The spin density isosurface (Figure 3c) shows than an intramolecular ET has happened in Int2', in which, interestingly, the spin density on the quinone ring being attacked localizes on the ortho- site of the carbon attacked by Nuc, and the conjugating carbonyl group only shares little spin. On the other hand, the spin density on the ring acting as the oxidant is delocalized over the two carbonyl groups.

After the formation of Int2', an intramolecular PT occurs through TS2, with a barrier of 17.0 $\mathrm{kcal} / \mathrm{mol}$. The evolution of the $\mathrm{C}-\mathrm{H}$ bond being broken and $\mathrm{O}-\mathrm{H}$ bond being formed along the Intrinsic Reaction Coordinate (IRC) of TS2 is shown in Figure 3b. The $\mathrm{O}-\mathrm{H}$ bond slowly decreases to $\sim 1.5$ angstrom in the pre-TS region (up to IRC step $\sim-5$ ), and is sharply shortened to $\sim 1.0$ angstrom through TS2. The change of $\mathrm{C}-\mathrm{H}$ bond length is asynchronous with the $\mathrm{O}-\mathrm{H}$ bond: it almost keeps unchanged at the pre-TS region, undergoes the first increase from IRC step -5 to 10 , and then another increase starting from IRC step 10. The two increases correspond to two phases of the overall reaction: the PT phase from IRC point -60 to 10, and then the hydroxyl rotation phase, which can be seen by comparing the geometry of step 10 (Figure 3b) and $\mathbf{2}(\mathrm{NaOMe}$ ) (Figure 3c). As for the spin density, all the points along the IRC are open-shelled, indicating that the reaction is almost a PT, although spin density at the ortho-carbon of both Int2' and TS2 is delocalized onto the two oxygen atoms in the final product 2 at the end of the reaction. According to the observations above: the presence of both Int2 and Int2', the PT-nature of TS2, and the presence of ET in Int2', it is concluded that the overall reaction follows a stepwise A-ET-PT mechanism when $\mathrm{NaOMe}$ acts as the Nuc.

\section{ACET-PT when Nuc $=\mathrm{Na}(\mathrm{THF})_{2}\left(\mathrm{OCOCF}_{3}\right)($ as noted by NaTFA $)$}

\section{Nuc $=$ NaTFA: The ACET - PT process}

(a)
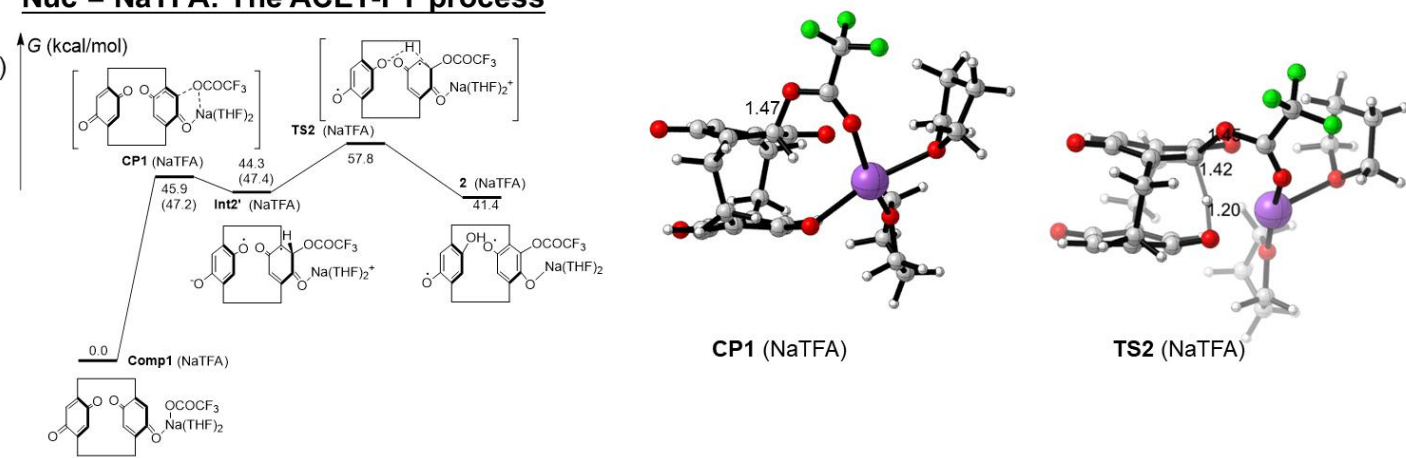

(b)

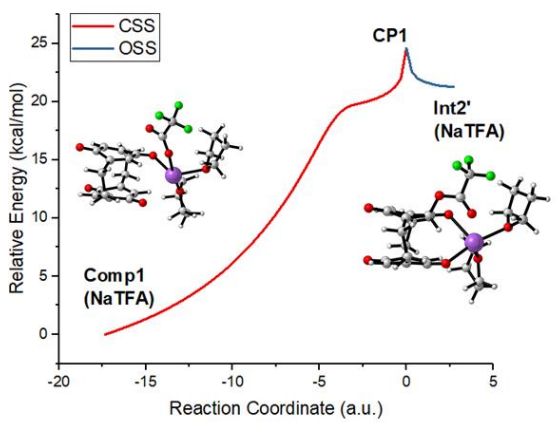

(c) CASSCF $(12,12)$ Optimized Orbitals of CP1
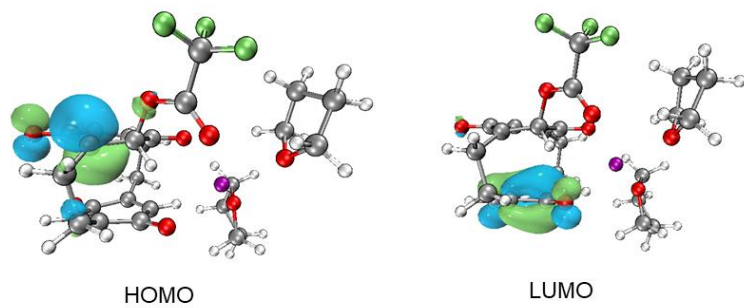

LUMO

Figure 4. (a) The Gibbs free energy profile for the ACET-PT reaction with $\mathrm{Na}(\mathrm{THF})_{2}\left(\mathrm{OCOCF}_{3}\right)$ 
(noted as NaTFA) as the nucleophile. The relative free energies at NEVPT2 $(12,12)$ level are shown in parentheses. The geometries of the key species are shown and distances are labelled in angstrom. (b) The downhill pathway starting from CP1 on the CSS and OSS potential energy surface. (c) The frontier orbitals of CP1 optimized at CASSCF(12,12)/def2-TZVPP level.

With NaTFA as Nuc, a tetrahedral intermediate-like compound Int2' also exists, as the case for $\mathrm{NaOMe}$, although it is much higher in energy ( $44.3 \mathrm{kcal} / \mathrm{mol}$ above the complex form by $\mathbf{1}$ and NaTFA). The ET-product Int2' undergoes a PT through TS2, with an overall barrier of 57.8 $\mathrm{kcal} / \mathrm{mol}$ to give the final product 2. Although the barrier is rather high (notably, there is no minimum corresponding to the addition product with 2,6-dimethyl-1,4-benzoquinone as the substrate, indicating that the ET resulted by the second benzoquinone ring is essential), causing the reaction experimentally inaccessible, it is still theoretically valuable, in providing mechanistic insights into the elementary reaction modes.

Although the formation of Int2' and the following PT are similar to the $\mathrm{NaOMe}$ case, there is one substantial difference: in the NaTFA case the close-shelled singlet (CSS) Int2 is no longer a minimum. By scanning the $\mathrm{C}-\mathrm{Nuc}$ distance (Supporting Information), the energy monotonously increases while NaTFA approaches on the CSS potential energy surface (PES). Instead, the addition product Int2' can only exists on the open-shelled singlet (OSS) PES. On the one hand, no transition state for the addition step was able to be found, on both CSS and OSS-PES. On the other hand, the crossing point between the CSS- and OSS-PES, CP1, at which the energy of the CSS and OSS state is degenerated, could be determined to be the critical point for the hopping from the CSS- to the OSS-PES. The downhill pathway starting from CP1, which is generated in a similar way to the Intrinsic Reaction Coordinate (IRC) and represents the reaction pathway across $\mathbf{C P 1}$, clearly shows that CP1 connects directly to 1Comp and Int2' on the CSS- and OSS-PES, respectively. As a result, it can be inferred that the addition step proceeds through CP1 instead of a transition state; once 1Comp goes across CP1, it will hop into the OSS-PES, and falls down the downhill pathway to afford Int2'. In this case, the addition and ET are coupled in one elementary step (ACET). The difference between ACET and stepwise ET-A-PT will be further discussed in the later section.

In addition to the DFT calculation, the multi-reference Complete Active Space SCF (CASSCF) and N-electron Valence State Perturbation Theory (NEVPT2) ${ }^{19}$ calculations were also performed, based on an active space with 12 orbitals and 12 electrons. The relative energy (shown in parentheses in Figure 4a) derived from NEVPT2 single point calculations were very close to the DFT results, supporting the reliability of the DFT results. According to the CASSCF-results, the electronic structure of the OSS state of CP1 is almost contributed ( 97\%) by the HOMO-LUMO excitation. optimized frontier orbitals (Figure 4c), the HOMO and LUMO distributes on the carbon atom orthoto the NaTFA attacked site and the quinone ring acting as the oxidant, respectively, which clearly reveals the existence of intramolecular single electron transfer.

\section{ACPCET when Nuc $=$ DMSO}




\section{Nuc = DMSO: the ACPCET process}

(a)

$\uparrow G(\mathrm{kcal} / \mathrm{mol})$

(b)

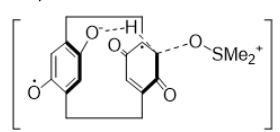

TS2 (DMSO)
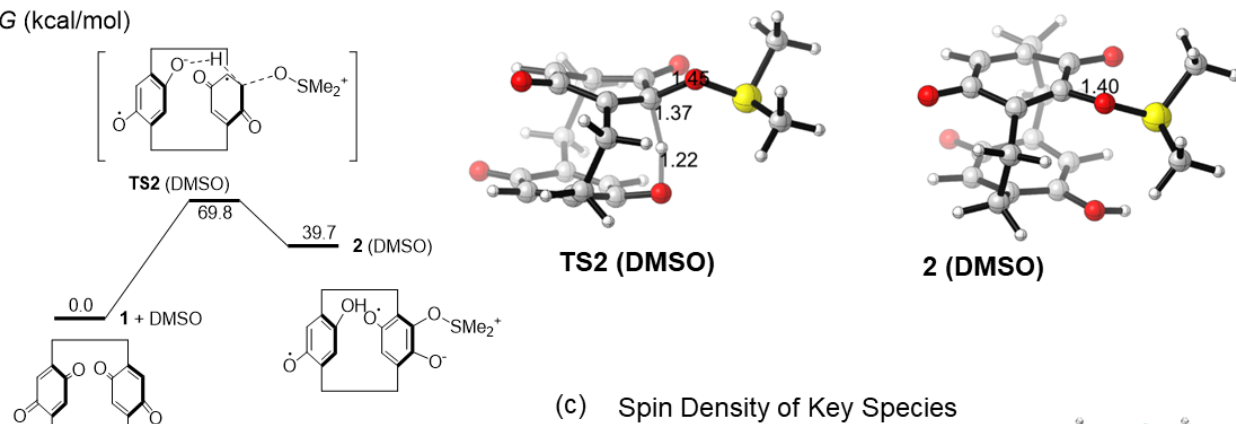

2 (DMSO)

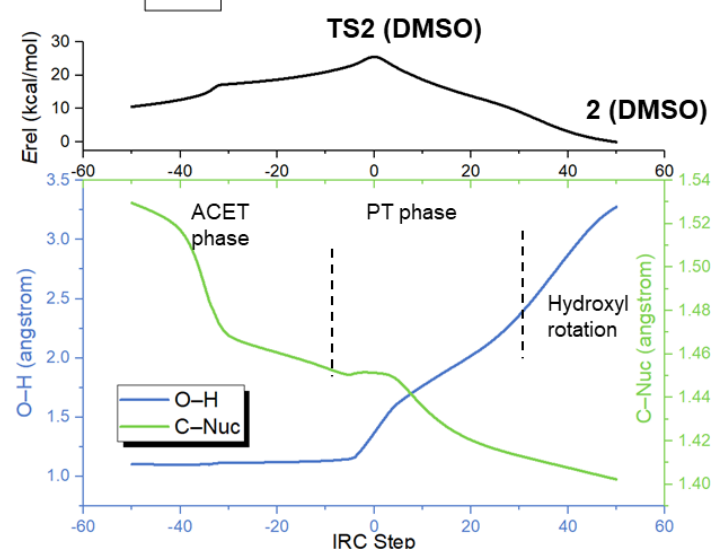

(c) Spin Density of Key Species

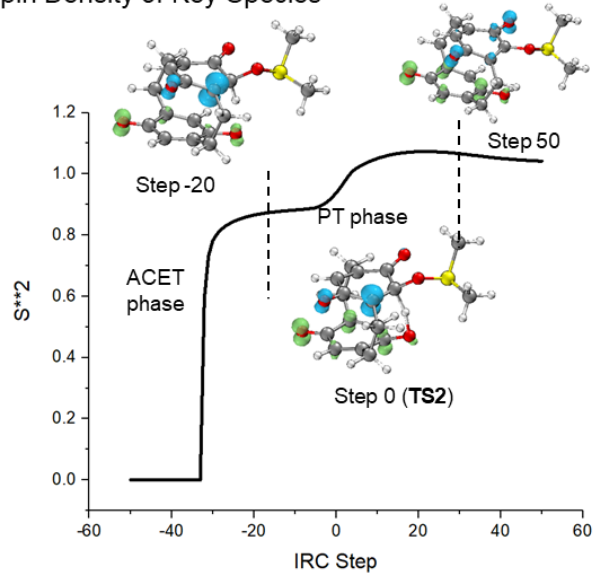

Figure 5. (a) The Gibbs free energy profile for the ACPCET reaction with DMSO (noted as NaTFA) as the nucleophile. (b) The evolution of key bond lengths along the IRC of TS2 (DMSO). (c) The $S^{* * 2}$ along the IRC, and spin density isosurface of selected points

As compared with NaTFA, DMSO is an even weaker Nuc, as reflected by its higher addition barrier (TS2 in Figure 5a) of $69.8 \mathrm{kcal} / \mathrm{mol}$, and absence of both Int2 and Int2' (Figure 2). The overall A-PT-ET process proceeds through only one transition state TS2 (DMSO). The IRC profile of TS2 (DMSO) can be divided into three stages: the approaching of DMSO to the substrate carbon atom accompanied by ET (the ACET phase, up to step -10 in Figure 5b), the PT phase (from step 10 to $\sim 30$ ), and the hydroxyl rotation phase. The complex remains close-shelled at the beginning of the addition phase, as seen by the $S^{* *} 2$ of zero in Figure $5 \mathrm{c}$; at step $\sim-30$, however, the ET event suddenly occurs, giving a $S^{* *} 2$ of $\sim 0.8$. Then the nucleophile continues approaching the substrate, although with a less slope, until it reaches $\sim$ step -10 , where the PT process starts according to the decreasing $\mathrm{O}-\mathrm{H}$ distance. Notably, although they are divided into different phases in order to magnify the asynchronousity of PT with ACET, the C-Nuc distance keeps decreasing after a short platform period in the PT phase, and finally reaches 1.40 angstrom in $\mathbf{2}$ (DMSO). The evolution of spin density along IRC shares a similar mode with the stepwise mechanism: first close-shelled, then ET event occurs although coupled with other bond formation and the ortho-carbon to the carbon being attacked accumulates spin density, and finally the spin is delocalized onto all the carbonyl groups in $\mathbf{2}$.

\section{To Distinguish the Mechanisms}


(a)

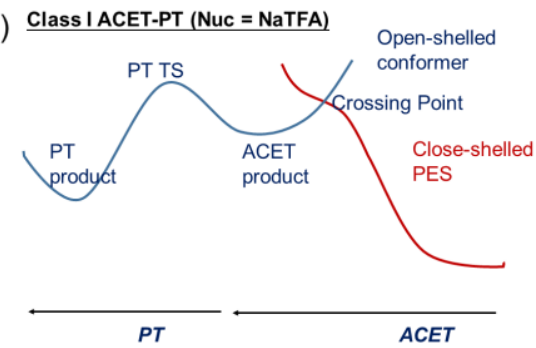

(b) Class II ACET-PT (not found in this work)

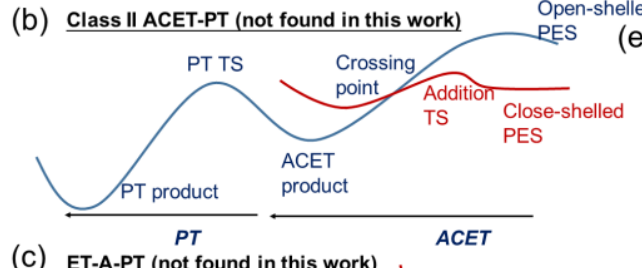

(c) ET
ET-A-PT (not found in this work)

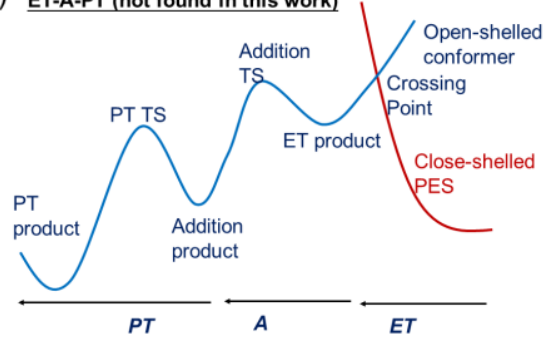

(d)
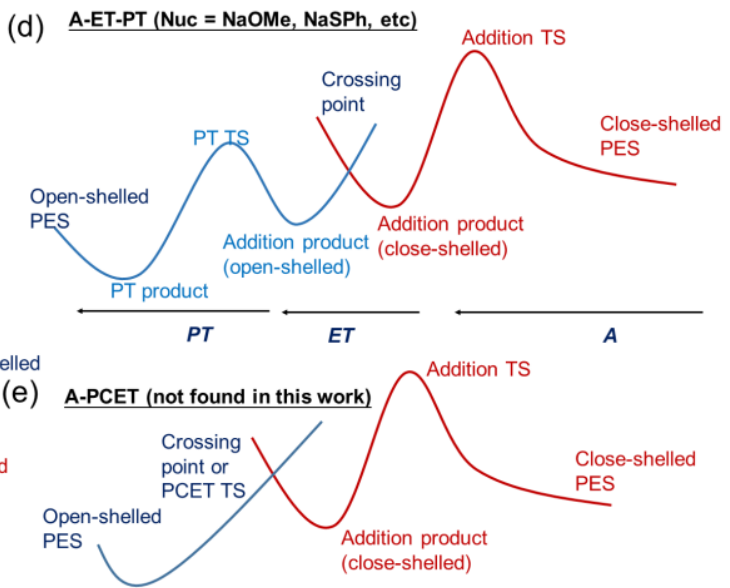

PT product

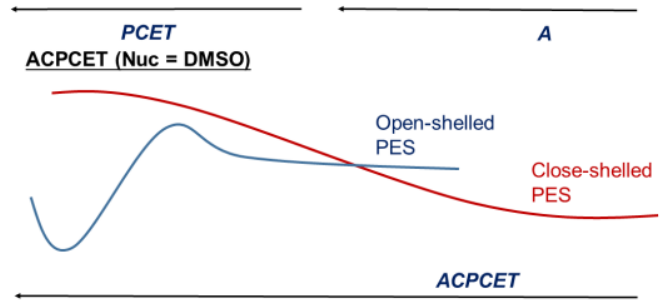

Figure 6. The schematic potential energy surface (PES) for the possible coupling modes among the

A, ET, PT reactions.

Up to now, we have discussed three examples with different coupling modes among A, ET, PT. In order to further clarify their relationship, the schematic models of their potential energy surfaces are plotted in Figure 6. Consider the crossing between the close-shelled and open-shelled PESs: in the presence of a strong nucleophile, the addition product Int2 is able to be a minimum on the CSSPES. In this regard, if the crossing point occurs later than its formation, and the resulted ET-product, namely open-shelled addition product Int 2' is also a minimum, then the reaction goes through two transition states divided by one crossing point, affording a typical stepwise A-ET-PT process (Figure 6d). If the ET product Int2' is not a minimum, and directly leads to the product $\mathbf{2}$, then the reaction follows an A-PCET mechanism (Figure 6e). On the other hand, if the crossing point appears earlier than the formation of Int2, an ET event should happen at the post-TS region of addition, and directly connects with the ACET product (Class II ACET, Figure 6b).

If the nucleophile is too weak to form a close-shelled addition product Int2 as a minimum, then we go the cases of Figure 6a, $6 \mathrm{c}$ and $6 \mathrm{f}$. The difference between ET-A-PT and Class I ACETPT relies on whether the "pure" ET product (namely $\mathbf{1}^{-}$and $\mathrm{Nuc}^{+}$) is able to exist as a minimum on the open-shelled surface; if a minimum is formed, then a barrier has to be overcome for addition, then a stepwise ET-A-PT reaction occurs (Figure 6c). However, in the Nuc = NaTFA case, there is no such ET product (see Table S1 for the redox potential), and thus no addition TS on the openshelled surface. Instead, after the ET event through the crossing point, the addition product with electron transferred is directly obtained, which is classified as the Class I ACET (Figure 6a). If even the ACET product Int2' cannot exist as a minimum, as seen for Nuc = DMSO case, only one transition state appears along the whole reaction, and gives a fully coupled ACPCET reaction (Figure 6f). 


\section{Quasiclassical Molecular Dynamics Simulation}

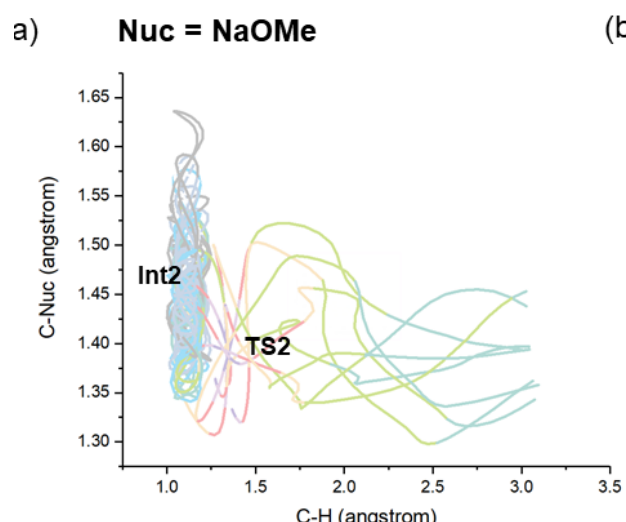

(b) Nuc $=$ DMSO
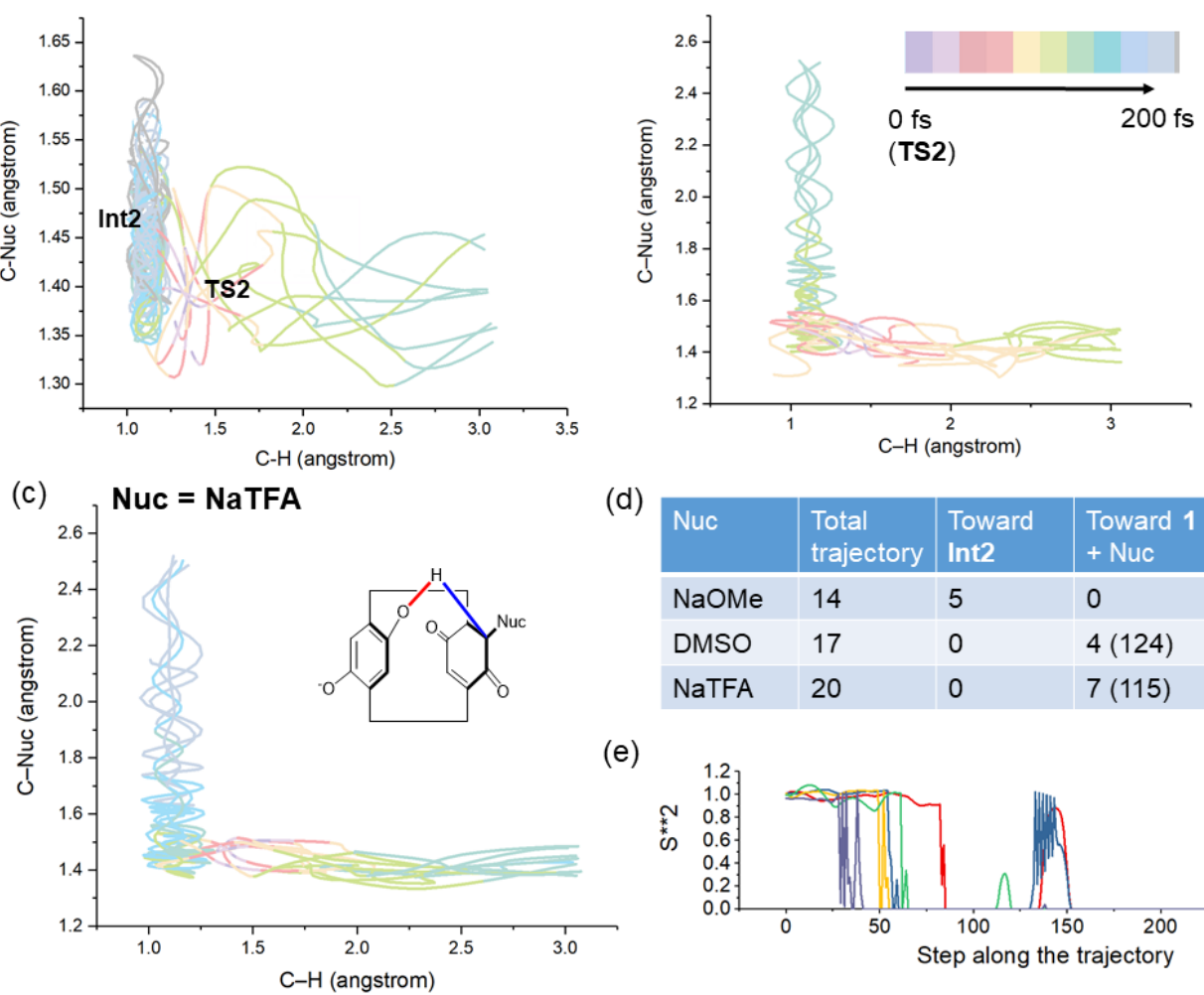

(d)

\begin{tabular}{|l|l|l|l|l|}
\hline Nuc & $\begin{array}{l}\text { Total } \\
\text { trajectory }\end{array}$ & $\begin{array}{l}\text { Toward } \\
\text { Int2 }\end{array}$ & $\begin{array}{l}\text { Toward } 1 \\
\text { + Nuc }\end{array}$ & Toward 2 \\
\hline NaOMe & 14 & 5 & 0 & $9(37)$ \\
\hline DMSO & 17 & 0 & $4(124)$ & $13(42)$ \\
\hline NaTFA & 20 & 0 & $7(115)$ & $13(35)$ \\
\hline
\end{tabular}

(e)

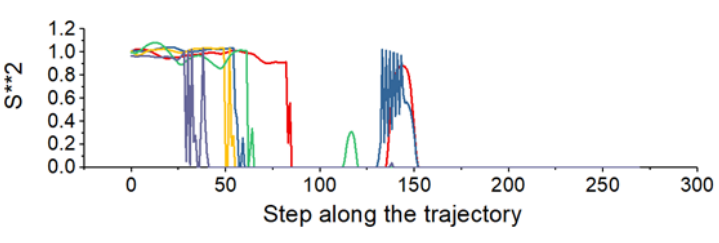

Figure 7. (a-c) The evolution of interested bond lengths along the trajectories initiated from TS2 with various nucleophiles. (d) The numbers of trajectories leading to each outcome. The average timing (fs) is shown in parenthesis. (e) The $S^{* * 2}$ along the trajectories leading to Int2 ( $\mathrm{NaOMe}$ ) from TS2 (NaOMe).

In order to further study the coupling among each step in real reaction, quasi-classical molecular dynamics trajectories were initiated from TS2 and the evolution of key bond lengths was recorded. For all the three nucleophiles, recrossing is quite common, as seen by the nearly doubled number of the trajectories leading to $\mathbf{2}$ over to Int $\mathbf{2}$ or separated $\mathbf{1}+$ Nuc. The PT event happens rapidly in $\sim 40 \mathrm{fs}$, regardless of the nucleophile. However, the fate of the trajectories toward the direction of Int2 (or Int2') is relevant to the nucleophile. On the one hand, for $\mathrm{NaOMe}, 5$ out of the 14 trajectories lead to close-shelled Int2, which is stable during the time period of $300 \mathrm{fs}$. The evolution of $S^{* * 2}$ for each point (Figure 7e) suggested that the complex returned to be close-shelled in almost $\sim 50$ fs, and then the system oscillates between Int2 $\left(S^{* * 2}=0\right)$ and Int2' $\left(S^{* * 2} \sim 0.8\right.$, appears near $\sim 150 \mathrm{fs}$ ). On the other hand, no Int2 or Int2' appears with adequate stability in the trajectories, and all the trajectories running toward their direction lead to dissociated $\mathbf{1}$ and Nuc in a similar timing of $\sim 120$ fs for NaTFA and DMSO. As a result, although they follow the ACET-PT and ACPCET mechanistic pattern respectively, dynamically they behave similarly: Int2' is not a "dynamically stable" intermediate, and the ACET and PT events are dynamically concerted in the NaTFA case. 


\section{Conclusion}

According to the discussions above, based on DFT and multireference calculations, IRC analysis and quasiclassical molecular dynamics, we have suggested the reaction mode of ACET and ACPCET, in which the addition, proton transfer and electron transfer steps effectively couple in one elementary step. With the [2.2] paracyclophane-derived biquinone $\mathbf{1}$ as the model substrate, we have shown that the mechanism of nucleophilic addition onto its $\mathrm{sp}^{2}$ carbon exhibits a consecutive change from stepwise A-ET-PT to ACET-PT and finally to ACPCET. The coupling of A with ET and PT is in consistence with the order of nucleophilicity: when the nucleophile is strong, a stepwise A-ETPT occurs; otherwise, with an adequately weak nucleophile, the ACET-PT, ACPCET, or other coupling modes has to occur to compensate the unfavorable addition, and which of them occurs depends on the shape of potential energy surface, as shown in Figure 6. Besides, quasiclassical trajectories show that these steps can be dynamically concerted, even when they seem to be stepwise according to the potential energy surface.

Although the examples of ACET and ACPCET in this work are of extremely high barrier, we believe that there exist other systems that ACET and ACPCET process is able to occur under experimentally accessible condition. For example, before submitting this work, we saw Fujii's latest report ${ }^{20}$ on the electron-coupled epoxidation reaction of olefin, with should be another example of ACET. Also, we believe that the remaining three types of coupling not discussed in this work, namely Class II ACET, A-PCET and ET-A-PT, can also be found in the future.

\section{Methods}

The Gaussian 16 package ${ }^{21}$ was employed to perform all the calculations, with the Gaussian 09 default integral grid. The wB97x-D functional ${ }^{22}$ was used for all calculations. For geometry optimization, the def2-SVP ${ }^{23}$ basis set was employed. Frequency calculations were followed to ensure stationary points were found, and to obtain Gibbs free energy correction at room temperature. Single point calculations were performed with the def2-TZVPP basis set. Both geometry optimization and single point calculation were performed under SMD implicit solvation ${ }^{24}$ of THF. The stability of wavefunction was checked for all the structures.

The spin density analysis were performed with the Multiwfn program ${ }^{25}$. The molecular geometry and isosurface were ploted with CYLView ${ }^{26}$ and $\mathrm{VMD}^{27}$.

The quasiclassical trajectory molecular dynamics simulations were performed using the PROGDYN program ${ }^{28}$. The initial geometry for each trajectory was generated by adding displacements that follows a QM-like Gaussian distribution to all vibrational modes higher than $10 \mathrm{~cm}^{-1}$ of TS2. Each real normal mode was given its zero-point energy plus a random Boltzmann sampling of the thermal energy available at $298.15 \mathrm{~K}$. Trajectories were propagated at wB97x-D/def2-SVP/SMD(THF) level in both the forward and backward directions, until the product formed or the length of trajectory is longer than $300 \mathrm{fs}$.

The multireference calculations were performed using the ORCA program ${ }^{29-30}$, with the natural orbital derived from the triplet state UHF calculations as the reference.

The crossing point between the CSS and OSS-PES was located by the KST48 program ${ }^{31}$.

\section{Acknowledgement}


Yumiao Ma thanks Hangzhou Yanqu Information Technology Co., Ltd for purchasing the license for Gaussian. Also thanks to all the students in Department of Chemistry, Tsinghua University, for their great love and encouragement toward Ma. Besides, Ma wants to mention a friend Zongchang Han (Tsinghua University); although he does not contribute in this work, he happened to have the concept of ACET in his independent project in 2018, although ACET did not occur in his project. In other words, Han has the idea of ACET earlier than Ma.

\section{References:}

1. Binstead, R. A.; Moyer, B. A.; Samuels, G. J.; Meyer, T. J., Proton-coupled electron transfer between [Ru (bpy) 2 (py) OH2] 2+ and [Ru (bpy) 2 (py) O] 2+. A solvent isotope effect (kH2O/kD2O) of 16.1. Journal of the American Chemical Society 1981, 103 (10), 2897-2899.

2. Huynh, M. H. V.; Meyer, T. J., Proton-coupled electron transfer. Chemical Reviews 2007, 107 (11), 5004-5064.

3. Mayer, J. M., Proton-coupled electron transfer: a reaction chemist's view. Annu. Rev. Phys. Chem. 2004, 55, 363-390.

4. Migliore, A.; Polizzi, N. F.; Therien, M. J.; Beratan, D. N., Biochemistry and theory of protoncoupled electron transfer. Chemical reviews 2014, 114 (7), 3381-3465.

5. Tyburski, R.; Liu, T.; Glover, S. D.; Hammarström, L., Proton-Coupled Electron Transfer Guidelines, Fair and Square. Journal of the American Chemical Society 2021, 143 (2), 560-576.

6. Warren, J. J.; Tronic, T. A.; Mayer, J. M., Thermochemistry of proton-coupled electron transfer reagents and its implications. Chemical reviews 2010, 110 (12), 6961-7001.

7. Weinberg, D. R.; Gagliardi, C. J.; Hull, J. F.; Murphy, C. F.; Kent, C. A.; Westlake, B. C.; Paul, A.; Ess, D. H.; McCafferty, D. G.; Meyer, T. J., Proton-coupled electron transfer. Chemical Reviews 2012, 112 (7), 4016-4093.

8. Hammes-Schiffer, S., Theoretical perspectives on proton-coupled electron transfer reactions. Accounts of chemical research 2001, 34 (4), 273-281.

9. Hammes-Schiffer, S.; Stuchebrukhov, A. A., Theory of coupled electron and proton transfer reactions. Chemical reviews 2010, 110 (12), 6939-6960.

10. Bruch, Q. J.; Connor, G. P.; Chen, C.-H.; Holland, P. L.; Mayer, J. M.; Hasanayn, F.; Miller, A. J., Dinitrogen Reduction to Ammonium at Rhenium Utilizing Light and Proton-Coupled Electron Transfer. Journal of the American Chemical Society 2019, 141 (51), 20198-20208.

11. Gentry, E. C.; Knowles, R. R., Synthetic applications of proton-coupled electron transfer. Accounts of chemical research 2016, 49 (8), 1546-1556.

12. Huang, L.; Ji, T.; Rueping, M., Remote Nickel-catalyzed cross-coupling arylation via protoncoupled electron transfer-enabled C-C bond cleavage. Journal of the American Chemical Society 2020, 142 (7), 3532-3539.

13. Lehnherr, D.; Lam, Y.-h.; Nicastri, M. C.; Liu, J.; Newman, J. A.; Regalado, E. L.; DiRocco, D. A.; Rovis, T., Electrochemical synthesis of hindered primary and secondary amines via proton-coupled electron transfer. Journal of the American Chemical Society 2019, 142 (1), 468-478.

14. Mora, S. J.; Odella, E.; Moore, G. F.; Gust, D.; Moore, T. A.; Moore, A. L., Proton-coupled electron transfer in artificial photosynthetic systems. Accounts of chemical research 2018, 51 (2), 445-453.

15. Roos, C. B.; Demaerel, J.; Graff, D. E.; Knowles, R. R., Enantioselective hydroamination of alkenes with sulfonamides enabled by proton-coupled electron transfer. Journal of the American Chemical 
Society 2020, 142 (13), 5974-5979.

16. Zhao, K.; Yamashita, K.; Carpenter, J. E.; Sherwood, T. C.; Ewing, W. R.; Cheng, P. T.; Knowles, R. R., Catalytic ring expansions of cyclic alcohols enabled by proton-coupled electron transfer. Journal of the American Chemical Society 2019, 141 (22), 8752-8757.

17. Ma, Y., Addition Coupled Electron Transfer (ACET) and Addition Coupled Electron Coupled Proton Transfer (ACPCET). ChemRxiv 2021.

18. Rebafka, W.; Staab, H. A., Ein „,intramolekulares Chinhydron” . Angewandte Chemie 1973, 85 (18), 831-832.

19. Angeli, C.; Cimiraglia, R.; Evangelisti, S.; Leininger, T.; Malrieu, J.-P., Introduction of n-electron valence states for multireference perturbation theory. The Journal of Chemical Physics 2001, 114 (23), 10252-10264.

20. Ishimizu, Y.; Ma, Z.; Hada, M.; Fujii, H., Rate-Limiting Step of Epoxidation Reaction of the Oxoiron(IV) Porphyrin $\pi$-Cation Radical Complex: Electron Transfer Coupled Bond Formation Mechanism. Inorganic Chemistry 2021.

21. Frisch, M. J.; Trucks, G. W.; Schlegel, H. B.; Scuseria, G. E.; Robb, M. A.; Cheeseman, J. R.; Scalmani, G.; Barone, V.; Petersson, G. A.; Nakatsuji, H.; Li, X.; Caricato, M.; Marenich, A. V.; Bloino, J.; Janesko, B. G.; Gomperts, R.; Mennucci, B.; Hratchian, H. P.; Ortiz, J. V.; Izmaylov, A. F.; Sonnenberg, J. L.; Williams; Ding, F.; Lipparini, F.; Egidi, F.; Goings, J.; Peng, B.; Petrone, A.; Henderson, T.; Ranasinghe, D.; Zakrzewski, V. G.; Gao, J.; Rega, N.; Zheng, G.; Liang, W.; Hada, M.; Ehara, M.; Toyota, K.; Fukuda, R.; Hasegawa, J.; Ishida, M.; Nakajima, T.; Honda, Y.; Kitao, O.; Nakai, H.; Vreven, T.; Throssell, K.; Montgomery Jr., J. A.; Peralta, J. E.; Ogliaro, F.; Bearpark, M. J.; Heyd, J. J.; Brothers, E. N.; Kudin, K. N.; Staroverov, V. N.; Keith, T. A.; Kobayashi, R.; Normand, J.; Raghavachari, K.; Rendell, A. P.; Burant, J. C.; Iyengar, S. S.; Tomasi, J.; Cossi, M.; Millam, J. M.; Klene, M.; Adamo, C.; Cammi, R.; Ochterski, J. W.; Martin, R. L.; Morokuma, K.; Farkas, O.; Foresman, J. B.; Fox, D. J. Gaussian 16 Rev. C.01, Wallingford, CT, 2016.

22. Chai, J.-D.; Head-Gordon, M., Long-range corrected hybrid density functionals with damped atomatom dispersion corrections. Physical Chemistry Chemical Physics 2008, 10 (44), 6615-6620.

23. Weigend, F.; Ahlrichs, R., Balanced basis sets of split valence, triple zeta valence and quadruple zeta valence quality for $\mathrm{H}$ to $\mathrm{Rn}$ : Design and assessment of accuracy. Physical Chemistry Chemical Physics 2005, 7 (18), 3297-3305.

24. Marenich, A. V.; Cramer, C. J.; Truhlar, D. G., Universal solvation model based on solute electron density and on a continuum model of the solvent defined by the bulk dielectric constant and atomic surface tensions. The Journal of Physical Chemistry B 2009, 113 (18), 6378-6396.

25. Lu, T.; Chen, F., Multiwfn: a multifunctional wavefunction analyzer. Journal of computational chemistry 2012, 33 (5), 580-592.

26. Legault, C., CYLview, 1.0 b. Université de Sherbrooke 2009, 436, 437.

27. Humphrey, W.; Dalke, A.; Schulten, K., VMD: visual molecular dynamics. Journal of molecular graphics 1996, 14 (1), 33-38.

28. Singleton, D. A.; Hang, C.; Szymanski, M. J.; Greenwald, E. E., A New Form of Kinetic Isotope Effect. Dynamic Effects on Isotopic Selectivity and Regioselectivity. Journal of the American Chemical Society 2003, 125 (5), 1176-1177.

29. Neese, F., Software update: the ORCA program system, version 4.0. Wiley Interdisciplinary Reviews: Computational Molecular Science 2018, 8 (1), e1327.

30. Neese, F.; Wennmohs, F.; Becker, U.; Riplinger, C., The ORCA quantum chemistry program 
package. The Journal of Chemical Physics 2020, 152 (22), 224108.

31. Ma, Y. KST48: A Powerful Tool for MECP location. https://github.com/RimoAccelerator/KST48 (accessed February 1). 